\title{
Protein import into chloroplasts: new aspects of a well-known topic
}

\author{
Anna Stengel, Jürgen Soll and Bettina Bölter* \\ Department of Botany, University of Munich, Menzinger \\ Str. 67, D-80638 Munich, Germany \\ ${ }^{*}$ Corresponding author \\ e-mail: boelter@|rz.uni-muenchen.de
}

\begin{abstract}
Protein import into plant chloroplasts is a fascinating topic that is being investigated by many research groups. Since the majority of chloroplast proteins are synthesised as precursor proteins in the cytosol, they have to be posttranslationally imported into the organelle. For this purpose, most preproteins are synthesised with an $\mathrm{N}$ terminal presequence, which is both necessary and sufficient for organelle recognition and translocation initiation. The import of preproteins is facilitated by two translocation machineries in the outer and inner envelope of chloroplasts, the Toc and Tic complexes, respectively. Translocation of precursor proteins across the envelope membrane has to be highly regulated to react to the metabolic requirements of the organelle. The aim of this review is to summarise the events that take place at the translocation machineries that are known so far. In addition, we focus in particular on alternative import pathways and the aspect of regulation of protein transport at the outer and inner envelope membrane.
\end{abstract}

Keywords: chloroplasts; protein import; redox mechanisms; regulation; Tic; Toc.

\section{Introduction}

Chloroplasts are the characteristic organelles of photosynthetic algae and plants. Their main function is the conversion of light into chemical energy by photosynthesis, but they also play essential roles in many other biosynthetic pathways, such as fatty acid biosynthesis, nitrite and sulphate reduction and amino acid biosynthesis. Chloroplasts - like mitochondria - originated from an endosymbiotic event, in which an ancestral photosynthetic cyanobacterium was engulfed by a mitochondriate eukaryotic host cell (Margulis, 1970). It is widely believed that this primary endosymbiosis occurred only once in plant evolution. Thus, all plastids - a family of organelles containing not only chloroplasts, but also leucoplasts, amyloplasts and chromoplasts - share a common ancestry. During evolution, the endosymbiont lost its autonomy through a massive transfer of genetic information from the prokaryotic genome to the host nucleus, although the organelle retained the competence for DNA replication, transcription and translation. To date, the chloroplast genome encodes approximately $50-150$ proteins, whereas the proteome is estimated to consist of 3500-4000 polypeptides (Leister, 2003). Due to their endosymbiotic origin, chloroplasts are surrounded by a double membrane. Consequently, the development of a protein import machinery became necessary for the relocation of proteins that are nuclear-encoded and synthesised in the cytosol, but located in the chloroplast. Organelle biogenesis and maintenance requires tight coordination of transcription, translation and protein import between the host cell and the organelle. Therefore, this review focuses on the different known ways of regulating protein import into chloroplasts. For studying protein translocation, the two model systems Pisum sativum and Arabidopsis thaliana are widely used. $P$. sativum is suitable for biochemical analysis, as large amounts of plant material and therefore of chloroplasts are easily generated. In contrast to this, since the genome of $A$. thaliana is fully sequenced, it is the system of choice for genetic studies.

\section{The Toc complex}

Most proteins are translocated into the chloroplast by the so-called general import pathway, mediated by the Toc (translocon at the outer envelope of chloroplasts) and the Tic (translocon at the inner envelope of chloroplasts) complexes (Figure 1; for a review see Soll and Schleiff, 2004; Bedard and Jarvis, 2005, and references therein). Translocation via this pathway is an energy-consuming process, requiring both ATP and GTP. The proteins responsible for preprotein recognition at the outer envelope are the two receptor components Toc159 and Toc34 (Kessler et al., 1994). Together with Toc75 (Schnell et al., 1994), they form the Toc core complex, a molecular machine of approximately $550 \mathrm{kDa}$, with a Toc75/Toc34/ Toc159 molecular stoichiometry of 4:4:1 (Schleiff et al., 2003a). Each translocon seems to be formed by a single central Toc159 molecule that is surrounded by four copies of Toc75/Toc34. Other components of the Toc complex are Toc64 (Sohrt and Soll, 2000) and Toc12 (Becker et al., 2004a).

Both Toc159 and Toc34 display homology to GTPases and are able to bind and hydrolyse GTP. They show high levels of similarity to each other within their GTPase domains. The proteins can also be phosphorylated, and (de)phosphorylation of Toc34 may regulate precursor recognition. In the model plant $A$. thaliana, most of the Toc components are encoded by small gene families. For Toc159, four homologues have been identified in the Arabidopsis genome, named atToc159, atToc132, atToc129 and atToc90. Expression data suggest that all of these 


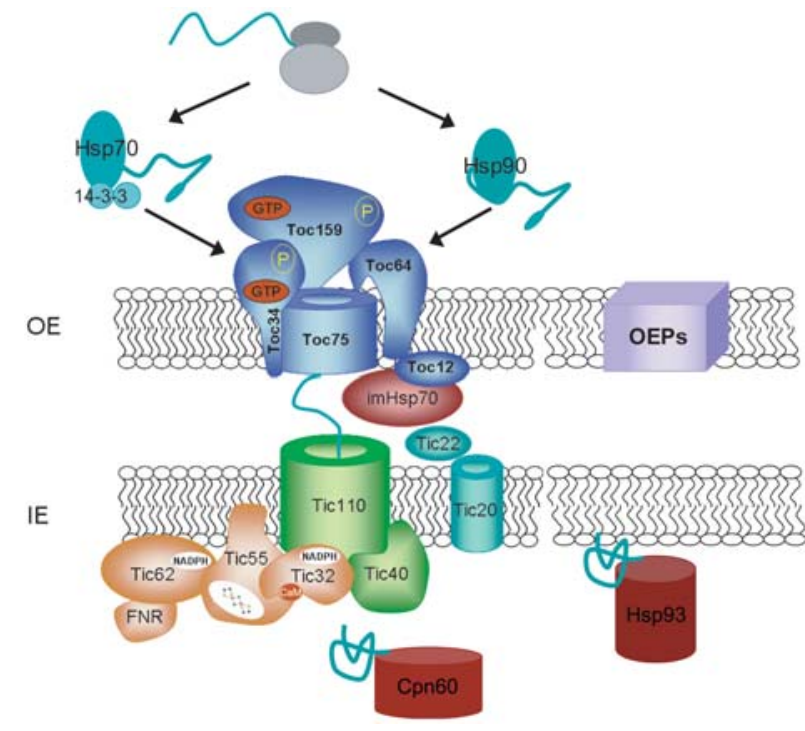

Figure 1 Model of protein import events and the components involved.

Preproteins destined for the chloroplast are translated on cytosolic ribosomes and interact with different molecular chaperones such as Hsp70/14-3-3 and Hsp90. They are targeted to the receptor components of the Toc complex (Toc34 and Toc159 or Toc64), which is followed by translocation through the Toc75 channel. In the intermembrane space, Toc12, imHsp70 and Tic22 interact with the translocation preproteins, handing them over to the Tic complex. Tic110 constitutes the channel of the inner envelope membrane and can also recruit stromal chaperones, probably with the help of Tic40. Tic32, Tic55 and Tic62 are most likely involved in mediating redox regulation of the import process. After translocation, preproteins are processed and folded, which might engage $\mathrm{Cpn60}$, or are further targeted to the inner envelope with the help of Hsp93.

genes are transcribed. The different homologues have more or less extensive deletions at the $\mathrm{N}$-terminus. Analysis of atToc159 mutants led to the suggestion that this protein is the predominant import receptor for photosynthetic chloroplast preproteins. It was also found to be expressed at higher levels compared to the other isoforms. In contrast, atToc132 and atToc120 seem to function in the import of non-photosynthetic proteins. Similar observations are true for the two Toc34 isoforms, atToc33 and atToc34: atToc33 seems to preferentially mediate the recognition of photosynthetic proteins, while atToc34 shows selectivity for the recognition of non-photosynthetic proteins instead. As co-immunoprecipitation experiments observed a preferential, though not exclusive interaction of atToc159 with atToc33 and atToc132/ 120 with atToc34, the existence of at least two different Toc subcomplexes was proposed (for a review see Bedard and Jarvis, 2005, and references therein) with different preferences for photosynthetic or non-photosynthetic proteins, respectively. A clear distinction between the roles of these putatively different complexes is, however, not yet known.

Toc75 forms the translocation channel of the Toc complex. Reconstitution assays in artificial bilayers showed that the protein is a cation-selective and voltage-dependent channel, with a pore size of $2-3 \mathrm{~nm}$. Like its functional equivalent Tom40 in the outer membrane of mitochondria, Toc75 is a $\beta$-barrel protein, formed by 18 amphiphilic $\beta$-strands (Schleiff et al., 2003a) and is deeply embedded in the outer envelope. Four homologues have been identified in the Arabidopsis genome, called atToc75-I, atToc75-III, atToc75-IV and atToc75-V. AtToc75-I was shown to be a pseudogene due to the presence of a retrotransposon insertion, and atToc75-IV is expressed only at a very low level. In addition, both have significant $\mathrm{N}$-terminal truncations. In contrast, atToc75-III and atToc75-V are abundant and strongly expressed. As atToc75-III is highly similar (73\% identity) to psToc75 throughout its mature sequence, it is believed that this protein is the general import pore of the Toc complex in Arabidopsis. AtToc75-V seems to represent the most ancestral form of a Toc75-like channel in chloroplasts, as phylogenetic analyses indicate that Toc75-V is more closely related to its prokaryotic ancestors than to Toc75 from plants (Eckart et al., 2002). Toc75 belongs to the Omp85 protein family. These proteins are believed to function as export pores and are found in bacteria and mitochondria. AtToc75- $\mathrm{V}$ could therefore take part in import or export of other macromolecules, or represent an alternative channel for chloroplast protein import. Another hypothesis proposes that atToc75-V is involved in insertion of $\beta$-barrel proteins into the outer envelope, a function also known for members of the Omp85 family.

Toc64 is characterised by two very different domains: at the $\mathrm{N}$-terminus, a domain similar to amidases is found, whereas the $\mathrm{C}$-terminus contains three tetratricopeptide repeat (TPR) motifs, which are exposed to the cytosol. TPR motifs are thought to be involved in protein-protein interactions and often mediate the interaction of proteins with molecular chaperones such as Hsp70 and Hsp90 (Frydman and Hohfeld, 1997). Thus, a role of Toc64 as a docking site for cytosolic chaperones interacting with precursor proteins was proposed, and recently this function of Toc64 for Hsp90-affiliated preproteins was demonstrated (Qbadou et al., 2006). Three homologous proteins have been identified in Arabidopsis. The most similar protein to psToc64 (67\% identity), atToc64-III, is associated with the chloroplast outer envelope, indicating that it is probably the functional orthologue of psToc64. In contrast, the truncated atToc64-I was not found in organelles. Interestingly, atToc64-V, which displays $52 \%$ identity to psToc64, is localised at the mitochondrial outer membrane. This led to the hypothesis that Toc64 may be specifically involved in the recognition of dual-targeted proteins (Chew and Whelan, 2004), which can end up in either chloroplasts or mitochondria. Furthermore, Toc64 was proposed as an alternative receptor for proteins lacking a cleavable transit peptide. An indication for this hypothesis can be found in mitochondria, where proteins with intrinsic targeting information are recognised by a different primary receptor (Tom20, and not Tom70 as usual; Truscott et al., 2003) that is structurally similar to Toc64.

The smallest subunit of the Toc complex is Toc12, an outer envelope protein exposing a soluble domain into the intermembrane space (IMS). Toc12 contains a Jdomain that interacts with a Hsp70 homologue in the IMS, stimulating its ATPase activity (Becker et al., 2004a). As Toc12 is associated with Toc64 and Tic22, these proteins might form a complex designed to assist in the transfer of precursor proteins across the IMS. 
Regulation of the Toc complex is mainly mediated by GTP/GDP binding and by phosphorylation of the receptor components Toc34 and Toc159 (for a review see Kessler and Schnell, 2004, and references within). In the cytosol, several chloroplast preproteins can be phosphorylated in the transit peptide by an ATP-dependent kinase (Waegemann and Soll, 1996; Martin et al., 2006). Phosphorylated peptides are then specifically recognised by 14-3-3 proteins, which, together with Hsp70, form a guidance complex that leads the preproteins to the Toc receptor. This might regulate chloroplast import at a cytosolic stage, as phosphorylation clearly influences the rate of translocation, although the targeting specificity is not altered (Waegemann and Soll, 1996). Therefore, precursor phosphorylation might be an important regulatory mechanism under certain developmental or environmental conditions that necessitates the preferential import of one precursor over the other. This could adapt and finetune protein import rates to the requirements of the plastid. The preprotein is then first recognised by Toc34, where binding of GTP to Toc34 increases the affinity for the transit peptide (Becker et al., 2004b). Furthermore, Toc34 has higher affinity for phosphorylated than for nonphosphorylated precursors, although phosphorylation is not essential for preprotein recognition by Toc34. Heterodimerisation of Toc34 with the GTP-binding domain of Toc159 might cause GDP/GTP exchange of the intrinsic GTPase. Alternatively, bound transit peptide may lead to the stimulation of GTPase activity, as preproteins can act as GTPase-activating factors for Toc34. The resulting GDP-Toc34 precursor complex has lower affinity for the preprotein than the GTP-bound form. This allows the preprotein to dissociate from the receptor, followed by transfer to Toc159-GTP. Dephosphorylation of the transit peptide is also thought to occur at this step, enhancing its dissociation from Toc34 and association with Toc159. Toc159 acts as the motor that facilitates movement of the preprotein through the translocation channel (Schleiff et al., 2003b). A new round of preprotein recognition and binding by Toc34 is provided by the exchange of GDP to GTP. On the other hand, Toc34 can be phosphorylated, which causes inactivation of the receptor, as neither preprotein recognition nor GTP binding can be mediated by phosphorylated Toc34. Reactivation via dephosphorylation is carried out by an ATP-dependent phosphatase (Sveshnikova et al., 2000).

\section{The Tic complex}

Translocation of proteins into chloroplasts also requires passing of the inner membrane, a process that is facilitated by the Tic complex. In most cases, proteins seem to translocate through the Toc and the Tic complexes simultaneously. Translocation across the inner envelope requires ATP hydrolysis, probably owing to the involvement of molecular chaperones in the stroma. In contrast to mitochondria, no membrane potential is needed for this translocation process.

To date, the Tic complex is thought to consist of at least seven proteins (for a review see Soll and Schleiff, 2004; Benz et al., 2007, and references therein). The most abundant subunit of the Tic complex is Tic110, which was also the first component to be identified (Lübeck et al., 1996). Tic110 consists of two domains: an $\mathrm{N}$-terminal domain with two predicted hydrophobic transmembrane $\alpha$-helices, and a hydrophilic C-terminal domain. Tic110 seems to be the central subunit of the Tic translocon and forms the translocation channel (Heins et al., 2002). Reconstitution assays into liposomes revealed that Tic110 is an ion channel with high conductance and a preference for cations. Electrophysiological data indicate a pore size of $15-20 \AA$, similar to that of the Toc channel Toc75, and large enough to allow the passage of an unfolded polypeptide chain. Furthermore, Tic110 is able to recruit stromal molecular chaperones (Cpn60 and ClpC) to the import site. Both chaperones may function in folding of imported proteins, but could also be part of an import motor, probably fixing the preprotein and preventing retrograde movement. Supporting the function of Tic110 as an important part of the Tic complex, T-DNA knockouts were shown to be embryolethal in the homozygous state.

Two other Tic components were found to be part of the Tic core complex together with Tic110: Tic55 and Tic62. The latter contains an NADPH binding site at the $\mathrm{N}$-terminus, and a repetitive module in the $\mathrm{C}$-terminal part (Küchler et al., 2002). These three proline-rich repeats specifically interact with ferredoxin:NADPH oxidoreductase (FNR), an enzyme that catalyses the final step within the electron transfer chain of oxygenic photosynthesis from ferredoxin to NADP, playing an essential role in $\mathrm{CO}_{2}$ fixation and chloroplast redox-controlled metabolism. A recent study identified Tic62 homologues in a variety of photosynthetic organisms, including not only plants, but also cyanobacteria and even green sulphur bacteria (Balsera et al., 2007). Interestingly, the full-length form of Tic62 was exclusively found in higher plants, whereas all non-vascular plants, including Physcomitrella patens, and all bacterial and algal species contained only a short, truncated form of Tic62 lacking the C-terminal part with the FNR-binding repeats. Thus, the ability to interact with FNR seems to be an evolutionarily new feature of Tic62, therefore probably providing a novel way of regulating chloroplast import in a redox-dependent manner. In contrast, the Nterminal part including the $\operatorname{NADP}(\mathrm{H})$-binding domain showed a high degree of conservation in all organisms analysed. This finding is also supported by other studies that revealed that Tic62 was partly (namely the N-terminal domain) of cyanobacterial origin (Reumann et al., 2005).

The other member of the Tic core complex, Tic55, was also described as a redox sensor protein (Caliebe et al., 1997). It contains a Rieske iron-sulphur centre and a mononuclear iron-binding site. Tic55 is predicted to contain two transmembrane helices, with the majority of the protein being exposed to the stroma. Database analysis revealed homology not only to plant oxygenases (PAO and Ptc52), but also to bacterial aromatic ring-hydroxylating dioxygenases, suggesting that Tic55 is of cyanobacterial origin. Rieske-type proteins are often involved in electron transfer, e.g., in the respiratory chain complexes, suggesting that Tic55 might play a role in redoxdependent regulation of protein import. 
Tic32 is the third candidate for mediating redox regulation of protein import into chloroplasts (Hörmann et al., 2004). It has been identified as an interaction partner of Tic110 and is a member of the conserved family of shortchain dehydrogenases (SDRs), characterised by an $\operatorname{NADP}(\mathrm{H})$-binding site at the $\mathrm{N}$-terminus (similar to Tic62), a $\beta$-sheet-stabilising motif in the central part (NNAG), and an active site at the $\mathrm{C}$-terminal end. Tic32 is strongly attached to the inner envelope and is essential for plant development, as homozygous knockout plants are embryo-lethal. The predicted dehydrogenase activity of Tic32 has been experimentally verified recently (Chigri et al., 2006), establishing Tic32 as a bona fide NADPHdependent dehydrogenase. Moreover, as Tic32 was identified as the predominant calmodulin-binding protein of the inner membrane, not only redox regulation but also calcium regulation (with calmodulin being the likely mediator) of chloroplast protein import could also be mediated by Tic32.

Tic40 shows clear homology to the co-chaperones Hip (Hsp70-interacting protein) and Hop (Hsp70 and Hsp90 organising protein). Furthermore, Tic40 contains a structure similar to TPR motifs. As already mentioned, these motifs are often involved in protein-protein interactions, especially with chaperones. Thus, Tic40 could act as a co-chaperone or be part of the Tic motor complex, together with Tic110, Hsp93 and ClpC. This is supported by the fact that the C-terminal Hip/Hop domain of Tic40 was shown to stimulate ATP hydrolysis of Hsp93, which dissociates from Tic40 in the presence of ADP, suggesting that Tic40 functions as an ATPase activation protein for Hsp93 (Chou et al., 2006). As Tic40 knockout plants do not show a drastic phenotype, the function of Tic40 does not seem to be essential for chloroplast import or plant development, but might rather represent a modulating subunit.

The only Tic component localised in the IMS is Tic22, which seems to be only loosely bound to the inner envelope. Together with Toc64, Toc12 and imHsp70, Tic22 is a member of an IMS complex (Becker et al., 2004a). The function of this complex, and therefore of Tic22, could be to coordinate the interaction of the Toc and Tic translocons, thus allowing efficient and direct translocation of preproteins through the IMS.

The smallest known subunit of the Tic complex is Tic20. It contains four predicted transmembrane domains and is deeply embedded in the inner envelope. Therefore, it has been suggested that Tic20 forms a translocation channel. This is supported by sequence analysis revealing a distant similarity to prokaryotic branched-chain amino acid transporters and to the mitochondrial channel proteins Tim23, Tim22 and Tim17 (Rassow et al., 1999). In several studies, Tic20 was demonstrated to interact with Tic22, but it was never found to interact with or was in close proximity to Tic110. If Tic20 and Tic110 are both preprotein-translocating channels, it does not seem likely that the two proteins are present in the same Tic complex. In contrast, they might rather work independently of each other, perhaps in different subcomplexes. Although the exact function of Tic20 needs further investigation, the importance of this protein for chloroplast viability becomes clear in Arabidopsis antisense lines, which show a pale to chlorotic phenotype and altered chloroplast ultrastructure. As already observed for several Toc components, Tic20 in Arabidopsis is encoded not by a single gene, but by a small gene family. Four homologues exist that are called atTic20-I, atTic20-II, atTic20-IV and atTic20-V. AtTic20-I shows the highest similarity to the pea protein (ca. 67\% identity); thus, atTic20-I and atTic20-IV (approx. 35\% identity) are considered as real orthologues and/or paralogues of Tic20 from $P$. sativum.

Recently, an additional component has been assigned to the Tic complex and called Tic21 (Teng et al., 2006). It was described as an integral membrane protein of the chloroplast inner envelope with four predicted transmembrane helices similar to Tic20. Nevertheless, the function of this protein as part of the Tic complex is questioned by another study, in which the same protein was identified as a metal permease (Duy et al., 2007).

\section{Processing and protein sorting in the stroma}

After translocation through the Toc and Tic complexes, the $\mathrm{N}$-terminal transit peptide has to be removed. This is achieved in an ATP-dependent manner by a large monomeric enzyme called stromal processing peptidase (VanderVere et al., 1995). Proteins that reach their final compartment in the stroma are further folded into an active conformation by stromal chaperones. However, for many proteins the stroma is not the final destination inside the chloroplast, as it contains not only a double envelope membrane, but also a third membrane system, the thylakoids, in which the photosynthetically active protein complexes are located. Beside these three different membrane systems, three aqueous sub-compartments can be distinguished, the IMS, the stroma and the thylakoid lumen. Therefore, effective intraplastidal sorting has to occur. Many proteins are targeted to the thylakoids, which are found exclusively in organisms performing oxygenic photosynthesis. In contrast to one general import pathway for protein import to the stroma, transport into or across the thylakoid membrane can be divided into at least four pathways: the Sec-dependent and the Tat-dependent pathways for transport of proteins into the thylakoid lumen, and the SRP-dependent and 'spontaneous' pathways for insertion of proteins into the thylakoid membrane (for a review see Jarvis and Robinson, 2004; Gutensohn et al., 2006, and references within). All four pathways have been identified for nuclear-encoded proteins, although transport of plastid-encoded proteins by the Sec and the SRP pathways is also described.

\section{General versus alternative import pathways}

The import of nuclear-encoded chloroplast preproteins through the Toc and Tic translocons described so far is known as the general import pathway. This is particularly true for stromal and thylakoid proteins. Nevertheless, some proteins also use alternative pathways that include other as yet unknown translocation machineries, vesicle transport via the endoplasmic reticulum (ER) and Golgi apparatus, and even spontaneous insertion into the outer 


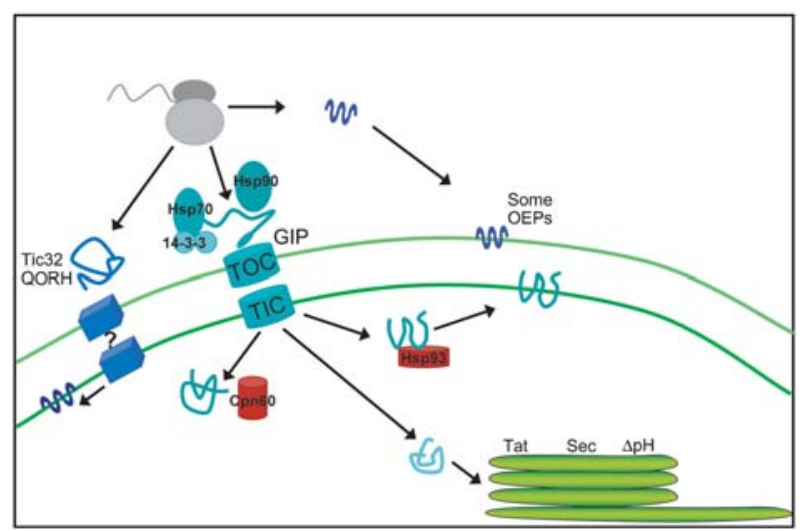

Figure 2 Schematic presentation of different import pathways. Most transit peptide containing preproteins engage the general import pathway (GIP) via Toc and Tic. Two inner membrane proteins, Tic32 and QORH, have been shown to use an alternative, as yet undefined import pathway. For some outer envelope proteins, spontaneous insertions have been observed, whereas others use the Toc translocon.

membrane (Figure 2). Very little is still known about these other ways of protein import into the chloroplast, and some of them have been discussed controversially over recent years. Interestingly, mainly proteins from the outer and inner chloroplast membrane seem to make use of these alternative pathways.

Proteins of the outer envelope are generally synthesised without an $\mathrm{N}$-terminal cleavable transit peptide. This includes not only most proteins of the Toc complex, but also outer envelope proteins such as OEP7, OEP16, OEP21, OEP24 and OEP37. These proteins are thought to insert into the membrane directly and spontaneously from the cytosolic side, independent of NTPs or proteinaceous components. The information for targeting and insertion into the outer membrane is localised within the mature part of these proteins. However, recent data suggest that outer envelope proteins might still use Toc75 as a translocation channel (Tu et al., 2004), but these Toc75 channels might work independently and are not associated with the receptor components. In particular, at Toc75-V might play a role in this insertion. Interestingly, one important exception of this group is Toc75 itself, which contains a bipartite transit peptide. The first part of this sequence functions in translocation via the general import pathway and is cleaved off by the stromal processing peptidase. The second portion of the signal functions as an intraorganellar targeting signal and is cleaved off in the IMS (for a review see Hofmann and Theg, 2005, and references therein).

In general, two pathways for protein targeting to the inner membrane are conceivable, both of which need the Toc/Tic system. First, hydrophobic stop-transfer signals within the mature part of the proteins cause lateral exit from the Tic channel into the membrane. Second, complete translocation into the stroma is followed by cleavage of the transit peptide and retargeting to the inner envelope membrane. The latter mechanism is characterised by the existence of a soluble intermediate, which was shown to occur in the case of Tic110 (Lübeck et al., 1996) and Tic40 (Li and Schnell, 2006). In addition, there are also some examples of inner envelope proteins lacking cleavable $\mathrm{N}$-terminal targeting signals (Miras et al., 2002; Nada and Soll, 2004): the chloroplast envelope quinone oxidoreductase homologue (ceQORH) and Tic32. These proteins carry the information for chloroplast targeting within their mature sequences, and Tic32 does not seem to need the general Toc/Tic import pathway at all. Nevertheless, the first ten $\mathrm{N}$-terminal amino acids were found to be essential for targeting. The only Toc/Tic component that is involved in this process is Tic22. This supports the hypothesis that Tic32 is inserted into the inner membrane directly from the IMS by an as yet unknown mechanism. Moreover, it has been proposed that the chlorophyll biosynthetic enzyme NADPH:protochlorophyllide oxidoreductase A (PORA) uses a novel alternative import complex containing OEP16, Toc34 and a Tic55 paralogue (Reinbothe et al., 2000). However, the existence of this pathway is controversially discussed (Philippar et al., 2007). These are only a few examples of import pathways of proteins without cleavable transit peptides, but it is conceivable that other pathways exist as well, since many nucleus-encoded chloroplast proteins without a cleavable transit peptide have been identified (Kleffmann et al., 2004). Therefore, a novel chloroplast-targeting pathway has been suggested, whereby it is proposed that proteins first enter the ER probably via an ER signal peptide, and are then transported to the chloroplast as vesicle cargo, which also involves the Golgi apparatus (Radhamony and Theg, 2006). It was suggested that this secretory pathway requires glycosylation in the $E R$, although the significance of glycosylation has not yet been investigated. The authors postulate three possible transport mechanisms to the stroma after vesicle fusion to the outer membrane: via an unknown transporter in the inner envelope, by passage through the Tic translocon independent of the Toc machinery, or by vesicle budding from the membrane itself. However, there is no proof of any of these possibilities so far, and a possible pathway from the ER to the plastid via the Golgi clearly needs further investigation. Another mechanism in which vesicles are involved is a transport system between the inner envelope and the outermost thylakoid membrane (for a review see Vothknecht and Soll, 2005, and references therein), which seems to be common in all vascular plants. As vesicle transport is a common phenomenon in the cytosol of eukaryotic cells, but is not described for prokaryotic organisms, it seems likely that chloroplast vesicle transport was created by transfer of a cytosolic system into the organelle. It was suggested that chloroplast vesicles could transport lipids from the inner envelope to the thylakoid membrane because the synthesis of membrane lipids occurs at the inner envelope. However, it cannot be excluded that other components such as proteins are also transported via these vesicles, as is widely used in the cytosol for protein transport.

\section{Regulation of protein import into chloroplasts}

Import of proteins into the chloroplast via both the Toc and Tic complexes needs to be regulated to allow reac- 
tion to the metabolic requirements of the plastid. At the Toc complex, regulation is facilitated by GTP/GDP binding and by phosphorylation, as discussed above (see chapter on the Toc complex). The identification of three Tic components that may be capable of sensing the redox state of the chloroplast led to the suggestion that chloroplast protein import may be regulated by redox signals (Figure 3). These three proteins are Tic55, Tic62 and Tic32 (Caliebe et al., 1997; Küchler et al., 2002; Hörmann et al., 2004). Tic55 contains a Rieske iron-sulphur centre and a mononuclear iron-binding site, both typically found in a specific class of bacterial oxygenases. These proteins often function in electron transfer reactions as electron acceptor or donor. Interestingly, diethylpyrocarbonate, a molecule known to disrupt Rieske centres, was found to inhibit the translocation of preproteins across the inner envelope into isolated chloroplasts, indicating that the Rieske centre of Tic55 might be important for protein import. The other two components that have been described as redox sensor proteins - Tic62 and Tic32 - both belong to the extended family of SDRs. Tic62 carries an NADPH-binding site at the N-terminus, which could also serve as an electron transfer site. The C-terminus was shown to interact with FNR. As FNR mediates the transfer of electrons from ferredoxin to NADP as the final step of electron transport during photosynthesis, it is possible that FNR is able to reduce the NADP molecule associated with Tic62. This might be only one step in a signal cascade to sense or react to the metabolic state of the chloroplast, as an electron transfer reaction from Tic62 to Tic55 could also occur. Interestingly, some electron carriers in the inner envelope have already been identified using electron paramagnetic resonance spectroscopy (Jäger-Vottero et al., 1997) and one of the proteins found in this study also contains an iron-sulphur centre, similar to Tic55. Whether this electron chain contains some of the Tic components possibly

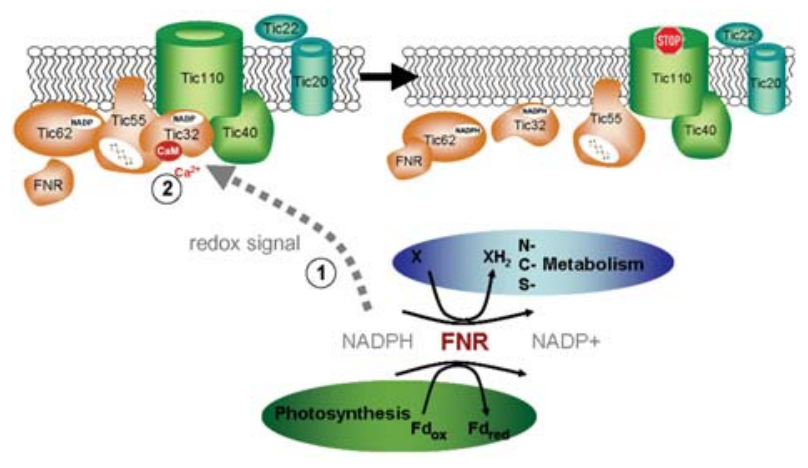

Figure 3 Regulation of protein import at the Tic complex. FNR plays a central role in reporting the redox and metabolic state of the chloroplast to the surrounding cell (1). Depending on the presence of $\mathrm{NADP}(\mathrm{H})$, the interaction with Tic components might be influenced, resulting in alteration of the Tic composition. Chloroplast import is also regulated by the second messenger calcium (2). Calmodulin (CaM) as a calcium sensor was found to interact with Tic32. This means that Tic32 is a potential transducer of the redox state, as well as of calcium regulation of protein import. So far, nothing is known about phosphorylation as another mode of protein import regulation at the Tic level. involved in redox-regulation remains elusive. Nevertheless, it is conceivable that conformational changes occur during the different reduction/oxidation steps, which may finally influence the gating behaviour of the pore Tic110 and therefore influence protein import. However, the ability of Tic62 to really transfer electrons has not been shown experimentally yet, in contrast to Tic32, which was recently identified as a bona fide NADPH-dependent dehydrogenase (Chigri et al., 2006). NADH could not substitute for NADPH, and the dehydrogenase activity of Tic32 is furthermore dependent on a lipid environment, a feature known for other SDRs. However, the in vivo substrates of the dehydrogenases and the sequence of any electron transfer in the Tic complex remain unknown. In addition, the results of this study indicate that NADP/ NADPH binding of Tic32 is important for its interaction with the Tic translocon, as the interaction of Tic32 - and of Tic62 - with Tic110 is significantly decreased in the presence of NADPH, a result that was not observed with NADP or NADH. Thus, by affecting the interaction between Tic110, Tic62 and Tic32, the NADP/NADPH ratio of the chloroplast could mediate the redox regulation of chloroplast protein import by influencing the assembly/disassembly of the complex. More evidence for redox regulation of protein translocation across the inner envelope is provided by a study in which different ferredoxin and FNR isoforms were differentially imported, depending on the redox state of the chloroplast. The authors used light or darkness to alter the state of the plastidic NADPH pool (Hirohashi et al., 2001). In the presence of light and active photosynthetic electron transport, the NADP/NADPH ratio is altered towards NADPH, which could then influence the selectivity of the import machinery. Such a regulatory mechanism is supported by the fact that deamino-NAD, a non-reducible NAD analogue, and ruthenium hexamine trichloride (HAR), an oxidant of $\mathrm{NAD}(\mathrm{P}) \mathrm{H}$, affected the in vitro import of two FNR isoforms differently (Küchler et al., 2002). The NADPH pool of chloroplasts is influenced not only by light conditions and photosynthetic electron transport, but also by the overall metabolic activity. Redox reactions involving $\mathrm{NADP}(\mathrm{H})$ dominate many enzymatic processes, and thus the availability of this compound reflects the metabolic state of the chloroplast, as well as the photosynthetic activity. Essential components such as amino acids and fatty acids are exclusively synthesised within chloroplasts, and thus the cellular requirements influence the plastidic status and performance. Therefore, exact fine tuning of cellular and chloroplastic functions and metabolism has to be maintained, and sensing of the plastidic redox state by components of a protein translocation machinery is a very elegant way to meet this challenge. The functionality of all plastids depends on the import of nuclear-encoded proteins, which has to be timed according to metabolic requirements; this is reflected by the redox state of the NADPH pool, which represents the lynchpin in sensing the status of the chloroplast (Figure 3).

Another possible mode of import regulation has been discovered recently. It was shown that chloroplast import is also influenced by calcium, an important second mes- 
senger in the cell. Calmodulin (CaM) was identified as the most likely candidate calcium sensor that could therefore play a role in calcium regulation of the chloroplast translocation process (Chigri et al., 2005). Furthermore, Tic32 was discovered as the predominant CaM-binding protein of the inner membrane, containing a CaM-binding domain at the C-terminal end. NADPH- and calciumdependent CaM binding to Tic32 appear to be mutually exclusive (Chigri et al., 2006). These results indicate that CaM regulation of import is conveyed by Tic32, making this protein the potential transducer of both redox and calcium regulation of protein import. Thus, Tic32 seems to constitute the sensor of redox signals from the inside and $\mathrm{Ca}^{2+}$ from the outside of the chloroplast, thereby representing the convergence of eukaryotic and prokaryotic signalling features. Upon high photosynthetic activity rendering the NADPH pool mostly reduced, the subcomplex consisting of at least Tic110, Tic62 and Tic32 disintegrates, which results in import inhibition of certain precursors (Hirohashi et al., 2001; Küchler et al., 2002). Under these conditions CaM cannot interact with Tic32 and calcium signalling might not be realisable. When Tic32 is not loaded with NADPH, CaM is able to bind and other precursors such as pSSU (Chigri et al., 2005) are imported to a lesser extent. Thus, it seems that by this type of bipartite regulation not only the import process in general is regulated, but perhaps also the formation of distinct subcomplexes that mediate the translocation of specific precursor proteins. Which kind of regulation dominates over the other and the way in which these very different methods of cellular regulation are coordinated remain an enigma and need intensive research.

\section{References}

Balsera, M., Stengel, A., Soll, J., and Bölter, B. (2007). Tic62: a protein family from metabolism to protein translocation. BMC Evol. Biol. 7, 43.

Becker, T., Hritz, J., Vogel, M., Caliebe, A., Bukau, B., Soll, J., and Schleiff, E. (2004a). Toc12, a novel subunit of the intermembrane space preprotein translocon of chloroplasts. Mol. Biol. Cell 15, 5130-5144.

Becker, T., Jelic, M., Vojta, A., Radunz, A., Soll, J., and Schleiff, E. (2004b). Preprotein recognition by the Toc complex. EMBO J. 23, 520-530.

Bedard, J. and Jarvis, P. (2005). Recognition and envelope translocation of chloroplast preproteins. J. Exp. Bot. 56, 22872320.

Benz, P., Bölter, B., and Soll, J. (2007). The role of the TIC machinery in chloroplast protein import. In: Molecular Machines Involved in Protein Transport Across Cellular Membranes, R. Dalbey, C. Koehler, and F. Tamanoi, eds. (New York, USA: Academic Press/Elsevier), in press, Chapter 17.

Caliebe, A., Grimm, R., Kaiser, G., Lubeck, J., Soll, J., and Heins, L. (1997). The chloroplastic protein import machinery contains a Rieske-type iron-sulfur cluster and a mononuclear iron-binding protein. EMBO J. 16, 7342-7350.

Chew, O. and Whelan, J. (2004) Just read the message: a model for sorting of proteins between mitochondria and chloroplasts. Trends Plant Sci. 9, 318-319.

Chigri, F., Soll, J., and Vothknecht, U.C. (2005). Calcium regulation of chloroplast protein import. Plant J. 42, 821-831.
Chigri, F., Hörmann, F., Stamp, A., Stammers, D.K., Bölter, B., Soll, J., and Vothknecht, U.C. (2006). Calcium regulation of chloroplast protein translocation is mediated by calmodulin binding to Tic32. Proc. Natl. Acad. Sci. USA 103, 1605116056.

Chou, M.L., Chu, C.C., Chen, L.J., Akita, M., and Li, H.M. (2006). Stimulation of transit-peptide release and ATP hydrolysis by a cochaperone during protein import into chloroplasts. J. Cell Biol. 175, 893-900.

Duy, D., Wanner, G., Meda, A.R., von Wiren, N., Soll, J., and Philippar, K. (2007). PIC1, an ancient permease in Arabidopsis chloroplasts, mediates iron transport. Plant Cell, in press, doi: 10.1105/tpc.106.047407.

Eckart, K., Eichacker, L., Sohrt, K., Schleiff, E., Heins, L., and Soll, J. (2002). A Toc75-like protein import channel is abundant in chloroplasts. EMBO Rep. 3, 557-562.

Frydman, J. and Hohfeld, J. (1997). Chaperones get in touch: the Hip-Hop connection. Trends Biochem. Sci. 22, 87-92.

Gutensohn, M., Fan, E., Frielingsdorf, S., Hanner, P., Hou, B., Hust, B., and Klösgen, R.B. (2006). Toc, Tic, Tat et al.: structure and function of protein transport machineries in chloroplasts. J. Plant Physiol. 163, 333-347.

Heins, L., Mehrle, A., Hemmler, R., Wagner, R., Küchler, M., Hörmann, F., Sveshnikov, D., and Soll, J. (2002). The preprotein conducting channel at the inner envelope membrane of plastids. EMBO J. 21, 2616-2625.

Hirohashi, T., Hase, T., and Nakai, M. (2001). Maize non-photosynthetic ferredoxin precursor is mis-sorted to the intermembrane space of chloroplasts in the presence of light. Plant Physiol. 125, 2154-2163.

Hofmann, N.R. and Theg, S.M. (2005). Chloroplast outer membrane protein targeting and insertion. Trends Plant Sci. $10,450-457$.

Hörmann, F., Küchler, M., Sveshnikov, D., Oppermann, U., Li, Y., and Soll, J. (2004). Tic32, an essential component in chloroplast biogenesis. J. Biol. Chem. 279, 34756-34762.

Jäger-Vottero, P., Dorne, A.J., Jordanov, J., Douce, R., and Joyard, J. (1997). Redox chains in chloroplast envelope membranes: Spectroscopic evidence for the presence of electron carriers, including iron-sulfur centers. Proc. Natl. Acad. Sci. USA 94, 1597-1602.

Jarvis, P. and Robinson, C. (2004). Mechanisms of protein import and routing in chloroplasts. Curr. Biol. 14, R1064-1077.

Kessler, F., Blobel, G., Patel, H.A., and Schnell, D.J. (1994). Identification of two GTP-binding proteins in the chloroplast protein import machinery. Science 266, 1035-1039.

Kessler, F. and Schnell, D.J. (2004). Chloroplast protein import: solve the GTPase riddle for entry. Trends Cell Biol. 14, 334-338.

Kleffmann, T., Russenberger, D., von Zychlinski, A., Christopher, W., Sjolander, K., Gruissem, W., and Baginsky, S. (2004). The Arabidopsis thaliana chloroplast proteome reveals pathway abundance and novel protein functions. Curr. Biol. 14, 354362.

Küchler, M., Decker, S., Hörmann, F., Soll, J., and Heins, L. (2002). Protein import into chloroplasts involves redox-regulated proteins. EMBO J. 21, 6136-6145.

Leister, D. (2003). Chloroplast research in the genomic age. Trends Genet. 19, 47-56.

Li, M. and Schnell, D.J. (2006). Reconstitution of protein targeting to the inner envelope membrane of chloroplasts. J. Cell Biol. 175, 249-259.

Lübeck, J., Soll, J., Akita, M., Nielsen, E., and Keegstra, K. (1996). Topology of IEP110, a component of the chloroplastic protein import machinery present in the inner envelope membrane. EMBO J. 15, 4230-4238.

Margulis, L. (1970). Origin of eukaryotic cells (New Haven, CT, USA: Yale University Press).

Martin, T., Sharma, R., Sippel, C., Waegemann, K., Soll, J., and Vothknecht, U.C. (2006). A protein kinase family in Arabidop- 
sis phosphorylates chloroplast precursor proteins. J. Biol. Chem. 281, 40216-40223.

Miras, S., Salvi, D., Ferro, M., Grunwald, D., Garin, J., Joyard, J., and Rolland, N. (2002). Non-canonical transit peptide for import into the chloroplast. J. Biol. Chem. 277, 4777047778.

Nada, A. and Soll, J. (2004). Inner envelope protein 32 is imported into chloroplasts by a novel pathway. J. Cell Sci. 117, 3975-3982.

Philippar, K., Geis, T., Ilkavets, I., Oster, U., Schwenkert, S., Meurer, J., and Soll, J. (2007). Chloroplast biogenesis: the use of mutants to study the etioplast-chloroplast transition. Proc. Natl. Acad. Sci. USA 104, 678-683.

Qbadou, S., Becker, T., Mirus, O., Tews, I., Soll, J., and Schleiff, E. (2006). The molecular chaperone Hsp90 delivers precursor proteins to the chloroplast import receptor Toc64. EMBO J. $25,1836-1847$.

Radhamony, R.N. and Theg, S.M. (2006). Evidence for an ER to Golgi to chloroplast protein transport pathway. Trends Cell Biol. 16, 385-387.

Rassow, J., Dekker, P.J., van Wilpe, S., Meijer, M., and Soll, J. (1999). The preprotein translocase of the mitochondrial inner membrane: function and evolution. J. Mol. Biol. 286, 105120.

Reinbothe, S., Mache, R., and Reinbothe, C. (2000). A second, substrate-dependent site of protein import into chloroplasts. Proc. Natl. Acad. Sci. USA 97, 9795-9800.

Reumann, S., Inoue, K., and Keegstra, K. (2005). Evolution of the general protein import pathway of plastids. Mol. Membr. Biol. 22, 73-86.

Schleiff, E., Soll, J., Küchler, M., Kuhlbrandt, W., and Harrer, R. (2003a). Characterization of the translocon of the outer envelope of chloroplasts. J. Cell Biol. 160, 541-551.

Schleiff, E., Jelic, M., and Soll, J. (2003b). A GTP-driven motor moves proteins across the outer envelope of chloroplasts. Proc. Natl. Acad. Sci. USA 100, 4604-4609.
Schnell, D.J., Kessler, F., and Blobel, G. (1994). Isolation of components of the chloroplast protein import machinery. Science 266, 1007-1012.

Sohrt, K. and Soll, J. (2000). Toc64, a new component of the protein translocon of chloroplasts. J. Cell Biol. 148, 12131221.

Soll, J. and Schleiff, E. (2004). Protein import into chloroplasts. Nat. Rev. Mol. Cell. Biol. 5, 198-208.

Sveshnikova, N., Soll, J., and Schleiff, E. (2000). Toc34 is a preprotein receptor regulated by GTP and phosphorylation. Proc. Natl. Acad. Sci. USA 97, 4973-4978.

Teng, Y.S., Su, Y.S., Chen, L.J., Lee, Y.J., Hwang, I., and Li, H.M. (2006). Tic21 is an essential translocon component for protein translocation across the chloroplast inner envelope membrane. Plant Cell 18, 2247-2257.

Truscott, K.N., Voos, W., Frazier, A.E., Lind, M., Li, Y., Geissler, A., Dudek, J., Muller, H., Sickmann, A., Meyer, H.E., et al. (2003). A J-protein is an essential subunit of the presequence translocase-associated protein import motor of mitochondria. J. Cell Biol. 163, 707-713.

Tu, S.L., Chen, L.J., Smith, M.D., Su, Y.S., Schnell, D.J., and Li, H.M. (2004). Import pathways of chloroplast interior proteins and the outer-membrane protein OEP14 converge at Toc75. Plant Cell 16, 2078-2088.

VanderVere, P.S., Bennett, T.M., Oblong, J.E., and Lamppa, G.K. (1995). A chloroplast processing enzyme involved in precursor maturation shares a zinc-binding motif with a recently recognized family of metalloendopeptidases. Proc. Natl. Acad. Sci. USA 92, 7177-7181.

Vothknecht, U.C. and Soll, J. (2005). Chloroplast membrane transport: interplay of prokaryotic and eukaryotic traits. Gene 354, 99-109.

Waegemann, K. and Soll, J. (1996). Phosphorylation of the transit sequence of chloroplast precursor proteins. J. Biol. Chem. $271,6545-6554$. 